\title{
Technologievorsprung in der Niederschlagsmessung
}

\author{
Kurt Nemeth, BDM Meteorology, OTT MESSTECHNIK GmbH \& Co. KG, Kempten (D)
}

\section{Zusammenfassung}

OTTMESSTECHNIKgelangmitdem Pluvio $^{2}$ ein großer Fortschritt in der Entwicklung modernster Technologien für die Niederschlagsmessung. Im Vergleich zu früherer Technologie bot bereits die Anfangsversion des von OTT entwickelten Niederschlagsmessers dem Nutzer wesentlich größere Auflösung und Genauigkeit bei den verschiedensten Wetterverhältnissen. Die neueste Version des Gerätes konnte in ihrer Leistung weiter verbessert werden, gleichzeitig wurden die Kosten entscheidend gesenkt. Dieser Artikel veranschaulicht die Vorteile der neuen Technologie gegenüber Methoden, die seit Jahrhunderten verwendet werden.

\section{Geschichte}

Die ersten Aufzeichnungen über Niederschläge wurden etwa 500 v. Chr. von den alten Griechen gemacht. Hundert Jahre später verwendeten die Inder Schüsseln, um Regenmengen zu messen. Die Ergebnisse wurden mit der zu erwartenden Ernte korreliert und dienten als Berechnungsgrundlage für Landsteuern. Später verwendete man zylindrische Gefäße mit Skalen, um Niederschläge aufzufangen, und zeichnete die Daten täglich von Hand auf.

\section{Automatische Regenmesser}

Sir Christopher Wren lebte im 17. Jahrhundert in England. Er war Astronom, Geometer und darüber hinaus einer der größten englischen Architekten seiner Zeit. Bekanntheit erlangte er vor allem durch den Entwurf von 53 Londoner Gotteshäusern, darunter der St Paul's Cathedral. Eine seiner weniger bekannten Erfindungen machte er im Jahre 1662 - er entwarf einen Regenmesser, den man nicht täglich aufsuchen musste (um die Ergebnisse aufzuzeichnen) - den ersten Kippwaagen-Regenmesser der Welt. Der Vorteil dieser Technik bestand darin, dass der Niederschlag mithilfe eines durch ein Uhrwerk betriebenen Schreibers aufgezeichnet werden konnte, sodass Sir Christopher die Daten nicht jeden Tag von Hand notieren musste. Erstaunlicherweise arbeiten auch heute noch viele Niederschlagsmesser auf der ganzen Welt nach diesem Prinzip.

\section{Der Kippwaagen-Regen- messer (Tipping Bucket Raingauge (TBR))}

Der TBR besteht aus einem Trichter, der auf einen Zylinder aufgesetzt wird, welcher entweder in den Grund eingelassen oder stabil aufgestellt wird. Im Trichter wird der Niederschlag aufgefangen und in einen von zwei kleinen Behältern abgeleitet, die auf einer Achse balancieren. Bei einer bestimmten Niederschlagsmenge (normalerweise 0,1 oder $0,2 \mathrm{~mm}$ ) kippt diese Waage und ein elektrisches Signal wird an einen Schreiber oder an einen Datensammler gesandt. Durch diesen Vorgang wird der Behälter (so gut wie) vollständig entleert und der Prozess kann sich wiederholen.

Jeder, der schon mit einem Kippwaagen Regenmesser gearbeitet hat, kennt die Probleme bei der Datenerfassung:

1. Der Kippwaagen-Behälter kippt

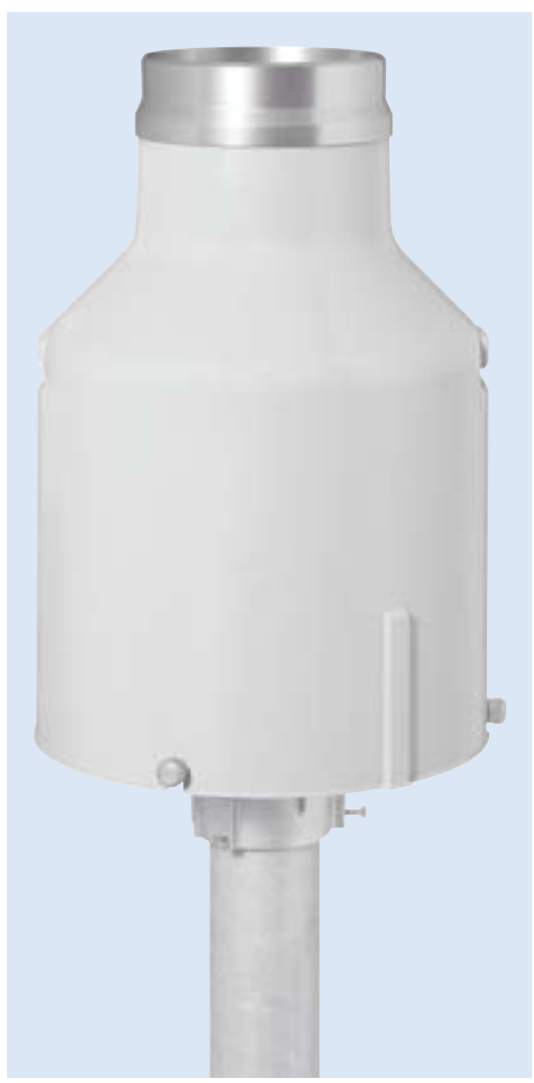

Abb. 1: Regenmesser Pluvio ${ }^{2}$ von OTT Messtechnik

erst in dem Moment, in dem er die erforderliche Niederschlagsmenge enthält. Das bedeutet, dass eine geringe Niederschlagsmenge, z. B. durch einen Nieselregen nicht aufgezeichnet wird, da der Behälter erst kippt, also einen Impuls auslöst, wenn zwischenzeitlich mehr Regen fällt. In der Aufzeichnung erscheint dann (fälschlicherweise) die Gesamtniederschlagsmenge $\mathrm{zu}$ diesem Zeitpunkt als ein Niederschlagsereignis.

2. Wenn der Behälter kippt, wird nicht der gesamte Inhalt ausgeleert. Eine kleine Wassermenge bleibt im Behälter, d. h., für den 


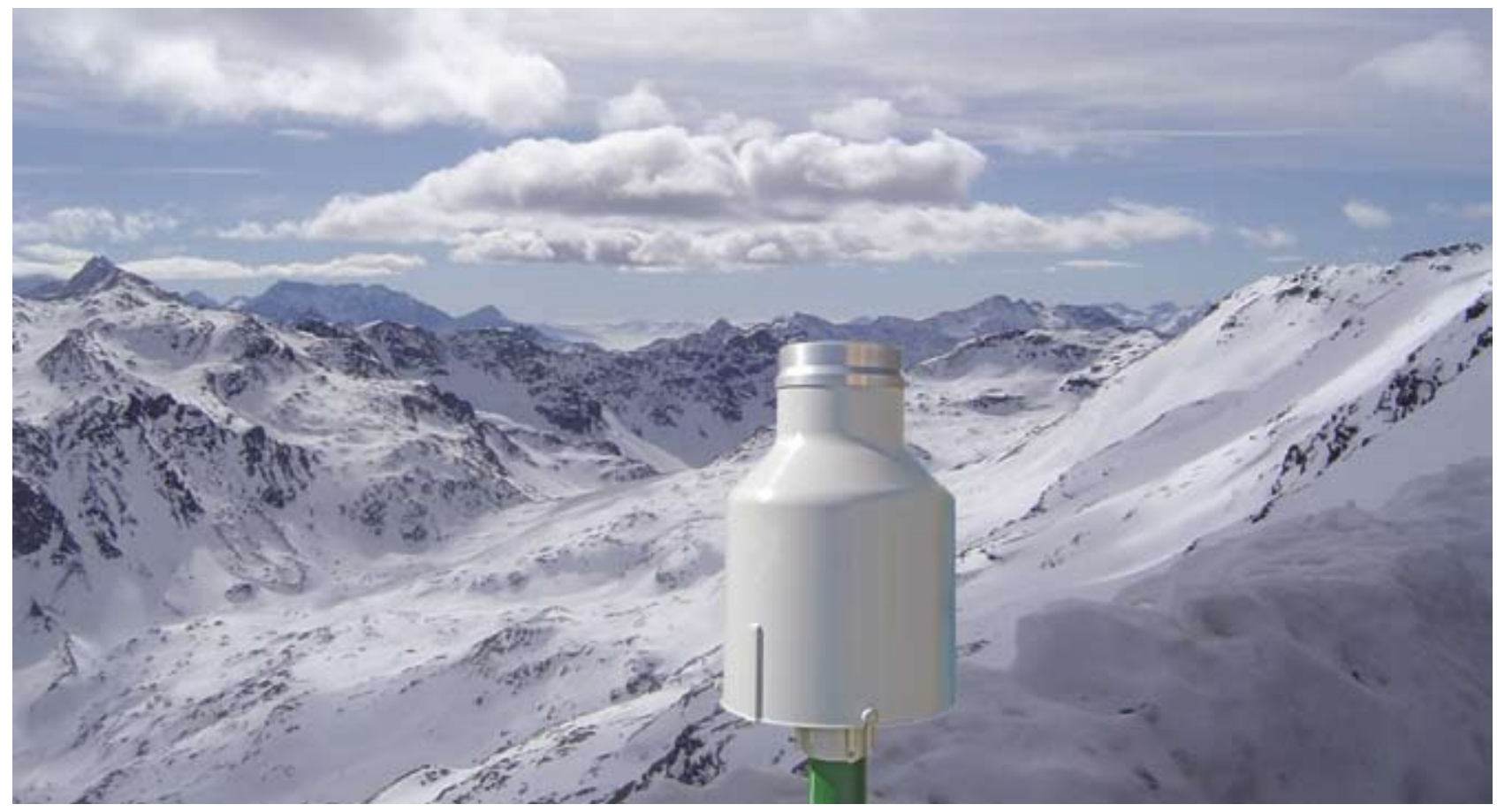

Abb. 2: Selbst bei Frost arbeitet die Technologie einwandfrei

nächsten Impuls wird weniger Niederschlag benötigt.

3. Schmutz- und Staubpartikel sammeln sich ebenfalls in dem Auffangbehälter und beeinflussen sowohl die Oberflächenspannung der Tropfen als auch das Volumen des Behälterinhalts, bis die Behälter gereinigt werden.

4. Oft werden Filter eingesetzt, um das Eindringen von Fremdpartikeln in die Behälter zu verhindern. Diese Filter verstopfen jedoch leicht, dies führt dann $\mathrm{zu}$ beträchtlichen Messfehlern.

5. Auch Wasser bleibt zu einem Teil im Trichter eines TBR zurück, verdunstet und verfälscht so ebenfalls die Messung.

6. In Zeiten mit starkem Niederschlag neigen Kippwaagen-Regenmesser dazu, zu geringe Werte abzulesen, da Wasser durch die plötzliche Kipp-Bewegung überschwappen kann. Auch geht beim Überfließen des Niederschlags von einem Behälter in den anderen Niederschlag verloren.

7. Eine weitere Einschränkung für den Einsatz von Kippwaagen-
Regenmessern ist es, dass sie nicht in der Lage sind, Schnee oder Hagel präzise zu messen, bzw. bei niedrigen Temperaturen überhaupt zu funktionieren. Dem kann durch den Einsatz einer Heizung abgeholfen werden, jedoch verdunstet durch die Heizung ein Teil des Niederschlags, bevor er gemessen wird. An entlegenen Orten stellt sich zudem meist das Problem der Stromversorgung.

Zusammenfassend lässt sich sagen, dass der Kippwaagen-Regenmesser im Jahre 1662 eine brillante Innovation war, die Meteorologen aber seit 300 Jahren nach einer Lösung suchen, um genauer und weniger arbeitsintensiv zu messen.

\section{Neue Technologie}

OTT MESSTECHNIK hat einen Regenmesser („Pluvio“) entwickelt, der die Probleme eines Kippwaagen-Regenmessers umgeht, indem der Niederschlag aufgefangen wird und die gesammelte Niederschlagsmenge mit einer hochpräzisen Wägezelle erfasst wird. Der Pluvio kam erstmals im Jahre 1994 auf den Markt; bis dato sind weltweit mehr als 5.000 Pluvios im Einsatz. Heute steht mit der zweiten Gerätegeneration, dem OTT Pluvio ${ }^{2}$, ein Gerät zur Verfügung, welches mit verbesserten Leistungsmerkmalen $\mathrm{zu}$ einem wesentlich günstigeren Preis als die erste Gerätegeneration angeboten werden kann. Dadurch ist er für eine weitaus größere Anzahl von Anwendungen attraktiv.

\section{OTT Pluvio²}

Der Hauptvorteil des OTT Pluvio² liegt darin, dass er bei allen Wetterbedingungen genau und zuverlässig misst. OTT Pluvio ${ }^{2}$ kann selbst das kleinste Niederschlagsereignis erfassen und zwar genau in dem Moment, in dem es stattfindet.

Die Messgenauigkeit des Gerätes wird durch intensiven Niederschlag in keiner Weise beeinflusst. Der Messbereich reicht von 0,1 mm bis zu der beeindruckenden Marke von $50 \mathrm{~mm} / \mathrm{min}$.

Selbst bei Frost arbeitet die Technologie einwandfrei. Im Auffangbehälter befindet sich ein 


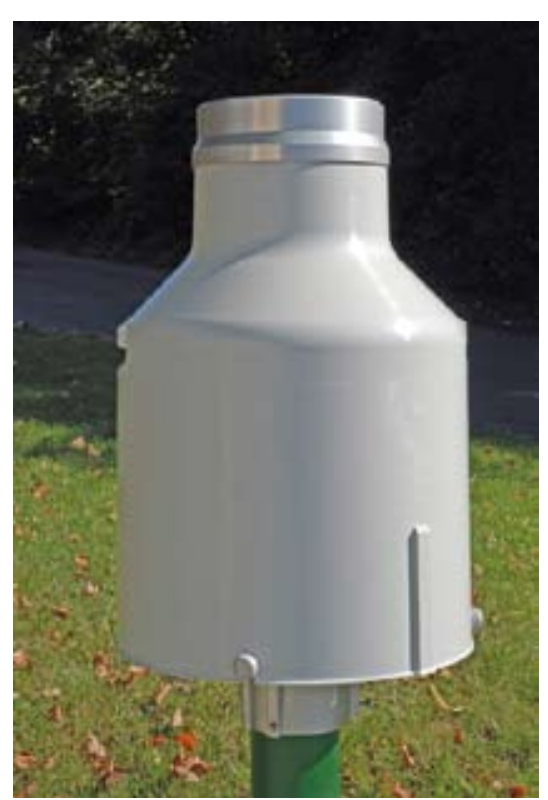

Abb. 3: Der Pluvio ${ }^{2}$ ist das Gerät der Wahl für Anwendungen, die Genauigkeit und Zuverlässigkeit unter allen Wetterbedingungen erfordern

Frostschutzmittel, welches die Ansammlung von Hagel und Schnee verhindert. Dadurch ist der OTT Pluvio $^{2}$ in der Lage, einen Messtemperaturbereich von -40 bis $+60{ }^{\circ} \mathrm{C}$ abzudecken.

Den OTT Pluvio ${ }^{2}$ gibt es mit zwei verschiedenen Auffangflächen: Die 200- $\mathrm{cm}^{2}$-Auffangfläche erfasst bis zu $1.500 \mathrm{~mm}$ Niederschlag und die 400- $\mathrm{cm}^{2}$-Auffangfläche hat eine Kapazität von $750 \mathrm{~mm}$. Diese hohe Kapazität übertrifft selbst den ursprünglich entwickelten Pluvio und verlängert die mögliche Messperiode zusätzlich.

Für die Version mit der kleineren Auffangfläche ist optional eine Ringheizung erhältlich, um die Bildung von Schneekappen zu verhindern.

Da während der Sommermonate ein Großteil des aufgefangenen Niederschlags verdunstet, verlängert sich die Messperiode entsprechend.

Ältere Technologien wie der Kippwaagen-Regenmesser verwenden manchmal eingebaute Heizungen mit Thermostat, um das Problem der Schnee-/Eisansammlung zu beheben. Dies führt aller- dings oft aufgrund des Auftauvorgangs zu Verzögerungen zwischen Ereignis und Messung und kann aufgrund des hohen Strombedarfs an entlegenen Messstellen recht unpraktisch sein.

Der OTT Pluvio ${ }^{2}$ dagegen arbeitet sehr sparsam - die Spannungsversorgung kann zwischen 9,6 und 28 V DC liegen, im Durchschnitt verbraucht er $12 \mathrm{~mA}$ bei $12 \mathrm{~V}$ DC.

Das Messsystem des OTT Pluvio $^{2}$ basiert auf einer hermetisch abgedichteten Wägezelle, mit Überlastschutz, direkt montiert auf der Wäge-Plattform. Eine hochentwickelte Elektronik und Auswertealgorithmen filtern mögliche windund temperaturbedingte Einflüsse aus. Jeder OTT Pluvio ${ }^{2}$ wird individuell temperaturkalibriert. Im Gegensatz zu Kippwaagen bleibt diese Kalibrierung für die gesamte Lebensdauer des Gerätes erhalten, so kann der Anwender sich auf lange Sicht und bei jedem Wetter auf die Genauigkeit seiner Daten verlassen. Die individuellen TemperaturKorrekturdaten sind elektronisch gespeichert.

Das zu messende Gewicht wird präzise ermittelt, mit ansteigender Genauigkeit von 2 Gramm entsprechend 0,10 $\mathrm{mm}$ Niederschlag und somit einer Auflösung von 0,01 mm. Die Wägezelle ist zudem unempfindlich gegenüber unsymmetrischer Beladung, d.h. zum Beispiel ungleichmäßige Verteilung von Schnee im Behälter wird ausgeglichen.

Der OTT Pluvio ${ }^{2}$ wurde von der Firma OTT MESSTECHNIK entwickelt und gebaut. Er entspricht der WMO-Richtlinie No. 8 und erfüllt höchste Qualitätsansprüche für eine lange Gerätelebensdauer. Die Betriebskosten sind minimal, da weder Kippwaagen-Behälter gereinigt noch verstopfte Trichter und Einlauffilter ausgetauscht werden müssen. Der OTT Pluvio² kommt mit einer Wartung pro Jahr aus. Die Kalibrierungsprüfung erfolgt mithilfe eines ISO-genormten Satzes von Kalibriergewichten.

Die Konfiguration des OTT Pluvio $^{2}$ erfolgt über eine USB-Schnittstelle, an die ein Pocket-PC oder Laptop/PC mit der Pluvio-Betriebssoftware angeschlossen wird. So hat der Benutzer jederzeit bequemen Zugang zu dem Gerät.

Auch für den Fall, dass im Feldeinsatz ein Schaden oder Fehler auftritt, haben die Pluvio-Entwickler eine Lösung gefunden: Die Elektronik mit Messeinheit kann einfach vor Ort ausgebaut und ausgetauscht werden. Dadurch wird das Risiko von Datenverlusten und hohen Wartungskosten erheblich verringert, da nicht das komplette Messgerät deinstalliert und in ein Servicecenter transportiert werden muss.

Viele Kunden besitzen bereits eigene Datensammler oder möchten den OTT Pluvio ${ }^{2}$ an eine bestehende Station anschließen. Hierfür sind standardisierte Schnittstellen verfügbar: Impuls, SDI-12 oder RS 485.

Viele OTT Kunden setzen den OTT Pluvio ${ }^{2}$ in bestehenden Messnetzen für Umweltdaten ein, wo weitere meteorologische oder hydrologische Sensoren, Datensammler, Energieversorgung und Datenübertragungstechnologie vorhanden sind. Solche Systeme können vor Auslieferung des OTT Pluvio² konfiguriert werden, sodass sofort mit dem Messen begonnen werden kann.

Als Spezialist für die Aufzeichnung von Umweltdaten bietet OTT eine ganze Reihe Strom sparender Datenlogger mit entsprechenden DFÜ-Technologien an; die Bandbreite reicht von Handhelds über GSM, GPRS, Funk- oder Satellitenübertragung.

\section{Anwendungen}

Der OTT Pluvio ${ }^{2}$ ist das Gerät der ersten Wahl für Anwendungen, die Genauigkeit und Zuverlässigkeit unter allen Wetterbedingungen 
erfordern. Besonders für sehr entlegene Messstellen, an denen häufige Besuche aufwendig und Zeit raubend sind, ist der Pluvio ${ }^{2}$ sehr beliebt. Dazu gehören nationale Überwachungs-Netzwerke für meteorologische Vorhersagen und Hochwasserwarnsysteme, Forschung, Landwirtschaft, Bewässerung, Flughäfen und vieles mehr.

\section{Die Zukunft}

Der OTT Pluvio ${ }^{2}$ wird sich zum Standard in der Niederschlagsmessung entwickeln und ältere Technologien ablösen. In Deutschland gilt er bereits als Maßstab für alle anderen Niederschlags-Messgeräte. In den Vereinigten Staaten hat der OTT Pluvio ${ }^{2}$ bei neuesten Tests verschiedener staatlicher Behör- den die Konkurrenz weit übertroffen; der nationale amerikanische Wetterdienst setzt den Pluvio auf wichtigen Flughäfen ein und der Geologische Dienst hat gerade eine umfangreiche Testreihe für das National Atmospheric Deposition Program (NADP) abgeschlossen, bei der es um die Entscheidung für den geeigneten Niederschlagsmesser für das National Trends Network geht.

Durch den günstigeren Preis für den OTT Pluvio ${ }^{2}$ werden nun viele Länder in der Lage sein, selbst größere mit Kippwaagen-Regenmessern ausgestattete Messnetze auf die neue Technologie umzurüsten. Wobei die Datenkontinuität gewährleistet bleibt.

Der OTT Pluvio ${ }^{2}$ ist die umfassendste Lösung für die autarke Er- fassung von genauen, zuverlässigen Echtzeit-Niederschlagsdaten, entwickelt von Experten mit langjähriger Erfahrung und Enthusiasmus für kompromisslose Niederschlagsmessung.

Sir Christopher Wren erfand den ersten Kippwaagen-Regenmesser, weil er die Notwendigkeit für automatische Messung erkannt hatte. Bei OTT sind wir überzeugt: Wenn er heute noch lebte - er wäre vom OTT Pluvio ${ }^{2}$ begeistert!

Informationen:

OTT MESSTECHNIK GmbH \& Co KG

Ludwigstraße 16

87437 Kempten

Deutschland

Tel. +49-831-5617-0

Fax +49-831-5617-209

info@ott.com

www.ott.com 